\title{
Status and perspectives of JUNO experiment
}

\author{
Agnese Giaz ${ }^{1, \dagger}$ \\ Università di Padova and INFN sezione di Padova \\ Via Marzolo, 8, Padova, Italy \\ E-mail: agnese.giazepd.infn.it
}

Precise measurements of the $\theta_{13}$ neutrino oscillation parameter by the Daya Bay, RENO and Double Chooz experiments, opened the path to the determination of the neutrino mass hierarchy. Indeed, whether the $v_{3}$ neutrino mass eigenstate is heavier or lighter than the $v_{1}$ and $v_{2}$ mass eigenstates it is one of the remaining undetermined fundamental aspects of the Standard Model in the lepton sector. Mass hierarchy determination will have an impact in the quest of the neutrino nature (Dirac or Majorana mass terms) towards the formulation of a theory of flavour. The Jiangmen Underground Neutrino Observatory (JUNO) is a large liquid scintillator neutrino detector under construction in the south of China. Thanks to the large 20 kton active mass and unprecedented energy resolution $(3 \%$ at $1 \mathrm{MeV})$ it will allow to determine the neutrino mass hierarchy with good sensitivity and to precisely measure the neutrino mixing parameters, $\theta_{12}$, $\Delta \mathrm{m}_{21}{ }^{2}$ and $\Delta \mathrm{m}_{\mathrm{ee}}{ }^{2}$ below the $1 \%$ level. Moreover, a large liquid scintillator detector will allow to explore physics beyond mass hierarchy determination. Indeed it will provide fundamental results on many topics in astroparticle physics, like supernova burst and diffuse supernova neutrinos, solar neutrinos, atmospheric neutrinos, geo-neutrinos, nucleon decay, indirect dark matter searches and a number of additional exotic searches. The paper will review the status of the experiment and give highlight on the possible physics research.

EPS-HEP2017, European Physical Society conference on High Energy Physics

5-12 July 2017

Venice, Italy 


\section{Introduction}

The future of neutrino oscillation studies foresees a rich and wide experimental program to determinate the elements of the Pontecorvo - Maki - Nakagawa - Sakata (PNMS) oscillation matrix with unprecedented accuracy, and to get more information in the features of neutrino properties. Therefore, the Dirac or Majorana nature of the neutrino mass term, mass hierarchy determination, octant of $\theta_{23}$, violating $\delta_{c p}$ phase and improved precision of the mass-mixing parameters (mixing angles as well as squared mass differences), are the core of the oscillation measurements.

Moreover, one fundamental aspect in the lepton sector of the standard model is if the $v_{3}$ neutrino mass eigenstate is heavier or lighter than the $v_{1}$ and $v_{2}$. We know that $m_{2}>m_{1}$ and $\left|\Delta \mathrm{m}_{31^{2}}\right|>>\left|\Delta \mathrm{m}_{21^{2}}\right|$ where $\Delta \mathrm{m}_{\mathrm{ij}}{ }^{2}=\mathrm{m}^{2}{ }^{2}-\mathrm{m}_{\mathrm{j}}^{2}$, but we don't know if $\mathrm{m}_{3}>\mathrm{m}_{1,2}$ or $\mathrm{m}_{3}<\mathrm{m}_{1,2}$. A schematic view of the normal and inverted mass hierarchy is shown in Fig. 1.

In this context, the JUNO experiment [1-2] will play a central role on two aspects: the determination of mass hierarchy and the precise measurements of the oscillation parameters $\left(\sin ^{2} \theta_{12}, \Delta \mathrm{m}_{21}{ }^{2}, \Delta \mathrm{m}_{\mathrm{ee}}{ }^{2}\right)$.

The electron antineutrino survival probability depends on the mass hierarchy even though the effect is small, but fortunately not negligible. The effect of the neutrino mass hierarchy on the electron antineutrino energy spectrum, measured at a medium-baseline of $\sim 50 \mathrm{~km}$, is shown in Fig. 1.
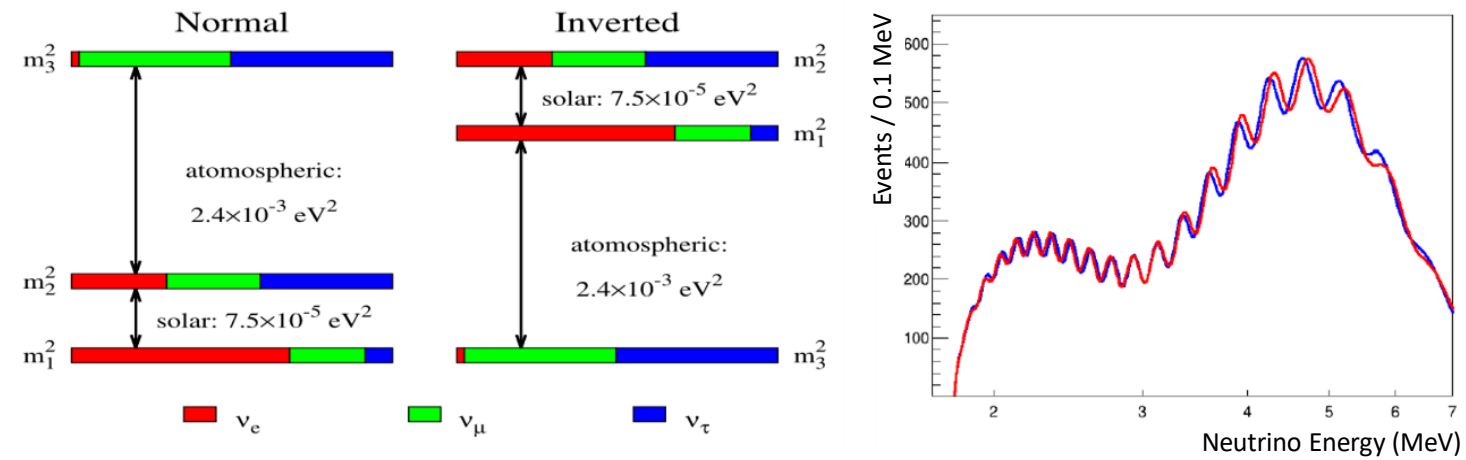

Figure 1: Left panel: a schematic view of the neutrino mass hierarchy: on the left the normal neutrino mass hierarchy scheme and on the right the inverted mass hierarchy one. Right panel: The electron antineutrino energy spectrum at a baseline of $50 \mathrm{~km}$, for both normal (red) and inverted (blue) hierarchy.

\section{The Jiangmen Underground Neutrino Observatory (JUNO) experiment}

The JUNO (Jiangmen Underground Neutrino Observatory) experiment is a reactor neutrino detector under construction in the south of China. The detector site was chosen with respect to optimal mean distance $(\sim 53 \mathrm{~km})$ from the cores of 2 nuclear power plants (Yangjiang 4 cores, and Taishan 6 cores) as shown in Fig. 2. The reactors produce a total thermal power of $35.8 \mathrm{GW}(26.6$ GW by the start of data taking around 2020). The $\bar{v}_{\mathrm{e}}$ flux will be detected through the reaction $\bar{v}_{e}+p \rightarrow e^{+}+n$ and its rate will be around 83 events per day. 


\subsection{The JUNO detector}

The JUNO detector main design goals are a large target mass, an excellent energy resolution and low energy scale uncertainty (less than $1 \%$ ). The central detector is composed by $\sim 20 \mathrm{kton}$ of active mass of LAB scintillator in a sphere of $35.4 \mathrm{~m}$ in diameter and it has unprecedented energy resolution of $3 \%$ at $1 \mathrm{MeV}$. The detector is overburden by about $700 \mathrm{~m}$ of rock.

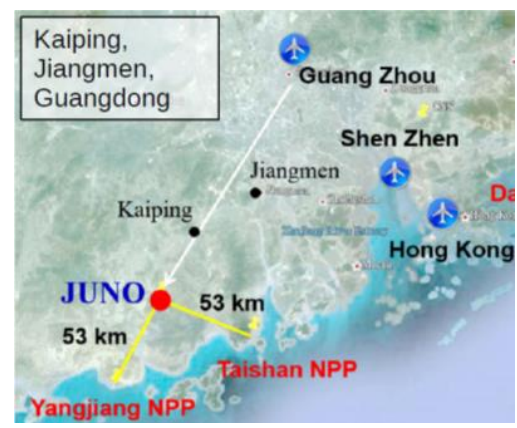

Figure 2: The JUNO detector site (indicated by a red point), is located inthe south of China. The two nuclear power plants at $\sim 53 \mathrm{~km}$ from JUNO experiment site are shown in the picture.

The sphere is enclosed by a water pool ( $44 \mathrm{~m}$ deep and $43.5 \mathrm{~m}$ high) that will be used as Cherenkov veto and as shield for environment radiation. The light is detected by two independent PMT systems, that having a coverage $>78 \%$ : the first PMT system is formed by 1800020 " PMTs, whereas the other one is formed by 25000 3" PMTs. The PMTs and the electronics will be under water. On the top of the water pool there is a solid scintillator as top tracker detector.

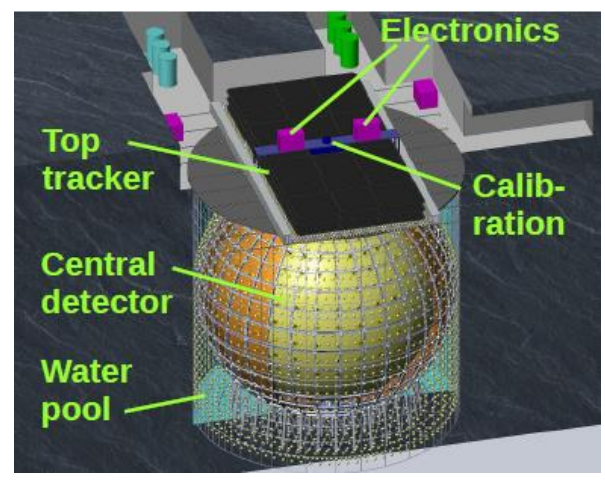

Figure 3: A schematic view of the JUNO detector. The main components are indicated on the scheme.

The JUNO experiment will have two independent calorimetry PMT systems. The requirements for the light collection system are: high optical coverage and high photon detection efficiency, acceptable noise level, good radio purity level, acceptable time resolution to achieve event reconstruction and a broad dynamic range. The large 20" PMT system will provide a coverage of about $75 \%$, while the small 3" PMT system a coverage of $3 \%$; that is why, the stochastic term will be around 3\% for the large PMTs and $14 \%$ for the small ones. The small PMTs will be faster and characterized by a better single p.e. resolution than the large ones and they will have a smaller dark noise with respect to the large PMTs. 
A feature of JUNO, with respect to previous large neutrino experiments is that the readout electronics will be integrated in the PMT housing to reduce the number of cables and noise. The front-end underwater electronics does not include only the analogue to digital conversion (ADC) stage but also intelligence of the electronics. A lot of functionalities will take place in a water proof box strictly coupled and sealed with the PMT. These are selective data readout, HV control, system monitoring, baseline control, signal conditioning and digitization, trigger primitive generation, segment buffering, calibration and synchronization tasks. For this reason, the electronics has to fulfill some constraints: it will be inaccessible after installation; the power consumption and the cost have to be minimized; the number of cables and water thigh connectors has to be minimized, too. The electronic prototype works and it was coupled to a PMT and the first data from a PMT were acquired. On the left panel of Fig. 3 there is a picture of the first electronic channel coupled to the PMT, while on the right panel there is an example of signals acquired with the set-up shown in the left panel.
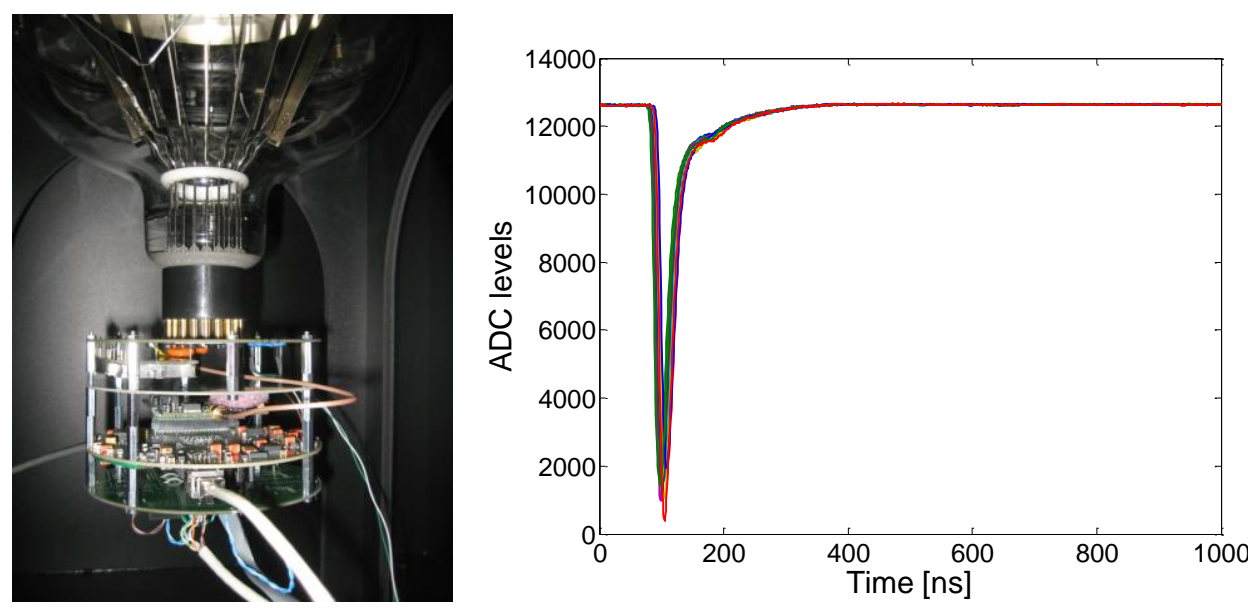

Figure 4: On left panel a compete electronic channel coupled to one PMT is shown. This set-up was used to acquire the first data, with the JUNO electronics. Some of the signals acquired are shown in the right panel.

\section{Physics with JUNO}

The main goal of the physics program with JUNO detector is the determination of the neutrino mass hierarchy with good sensitivity ( $3 \sigma$ after 6 years) and the precise measurement the neutrino mixing parameters. Indeed, the $\sin ^{2} \theta_{12}$ parameter is current know with a precision $4.1 \%$, $\Delta \mathrm{m}_{21}{ }^{2}$ with $2.3 \%$, and $\Delta \mathrm{m}_{\mathrm{ee}}{ }^{2}$ with $1.6 \%$. With the JUNO experiment we expect to measure all these parameters with a precision below $1 \%$.

Many other neutrino physics aspects can be studied with JUNO detector such as supernova burst and diffuse supernova neutrinos that will provide information on standard neutrino model; from solar neutrinos it is possible to get information about on ${ }^{7} \mathrm{Be}$ flux and ${ }^{8} \mathrm{~B}$ one; while from geo-neutrinos the information on U/Th ratio can be obtained. Moreover, JUNO will provide also information on the indirect dark matter searches and it will exploit physics of atmospheric neutrinos. Furthermore by studying the nucleon decay it will be possible to have information on proton decay $\left(p \rightarrow K^{+}+\bar{v}_{e}\right)$. 


\section{Summary}

JUNO is a multipurpose reactor neutrino experiment under construction in the south of China. The founding of JUNO project was approved in 2013 and the JUNO collaboration was officially formed in 2014. Now the civil construction, the detector component, PMT production and electronics development are in progress. The detector installation is foreseen for 2019 and the liquid scintillator filling and the data taking are foreseen for 2020.

The physics program of JUNO experiment involves the mass hierarchy determination on $3 \sigma$ after 6 years or even better. A significant improvement in the uncertainty of $\sin ^{2} \theta_{12}, \Delta \mathrm{m}_{21}{ }^{2}$ and $\Delta \mathrm{m}_{\mathrm{ee}}{ }^{2}$ will also be obtained. The JUNO experiment will provide also data from supernovae, solar, atmospheric and geo-neutrinos, proton decay and it will improve the knowledge on these fields.

\section{References}

[1] T. Adam et al. (JUNO Collaboration), “The JUNO Conceptual Design Report”, arXiv:1508.07166 (2016).

[2] F. An et al. (JUNO Collaboration), "Neutrino physics with JUNO”, Journal of Phys. G (Nucl. and Part. Phys.) 43, id. 030401 (2016). 